\title{
Objects that Cannot Be Taken Apart with Two Hands*
}

\author{
J. Snoeyink ${ }^{1}$ and J. Stolfi ${ }^{2}$ \\ ${ }^{1}$ Department of Computer Science, University of British Columbia, \\ Vancouver, BC V6T 1Z4, Canada \\ snoeyink@cs.ubc.ca \\ ${ }^{2}$ Computer Science Department, Universidade Estadual de Campinas, \\ Caixa Postal 6065, 13081 Campinas, SP Brazil \\ stolfidcc.unicamp.br
}

\begin{abstract}
It has been conjectured that every configuration $\mathscr{C}$ of convex objects in 3-space with disjoint interiors can be taken apart by translation with two hands: that is, some proper subset of $\mathscr{C}$ can be translated to infinity without disturbing its complement. We show that the conjecture holds for five or fewer objects and give a counterexample with six objects. We extend the counterexample to a configuration that cannot be taken apart with two hands using arbitrary isometries (rigid motions).
\end{abstract}

\section{Introduction}

Have you ever felt, when you were trying to put something together, that you needed an extra hand? In this paper we investigate questions of how many moving subassemblies are necessary to assemble (or by reversing time, disassemble) configurations of convex objects. These questions have applications in the fields of mechanical assembly planning, robotic manipulation, computer graphics, and recreational mathematics, as well as giving insight into the complexity of generalizing from the Euclidean plane to 3-space.

Let $\mathscr{C}$ be a finite set of convex sets (objects) in Euclidean space $E^{d}$. Objects in this paper always have disjoint interiors. We say that configuration $\mathscr{C}$ can be taken apart if some pair of objects $A, B \in \mathscr{C}$ can be moved arbitrarily far apart by rigid motions of the objects of $\mathscr{C}$ such that the objects always have disjoint interiors.

\footnotetext{
* The research of J. Snoeyink was supported in part by an NSERC Research Grant. J. Stolfi was previously at DEC Systems Research Center, Palo Alto, CA, USA.
} 
$\mathscr{C}$ can be taken apart with $k$ hands if it can be partitioned into $k$ sets, $\mathscr{C}=$ $\mathscr{C}_{1} \cup \cdots \cup \mathscr{C}_{k}$, and taken apart such that no relative motion occurs inside any $\mathscr{C}_{i}$. Finally, configuration $\mathscr{C}$ can be taken apart by translation if it can be taken apart using a finite sequence of translational motions.

In the plane any configuration of convex objects can easily be taken apart by translation with two hands. An aboveness relation can be defined for objects by saying that $A$ is above $B$ if a vertical ray drawn upward from a point in the interior of $B$ encounters $A$. This relation is acyclic in the plane [5], [6]-a fact that is useful in many algorithms [8], [15]. Thus, there is a maximum object with respect to aboveness and any maximum object can be translated upward to infinity. Computer graphics textbooks often illustrate that the aboveness relation for convex objects in 3-space can have cycles: three triangles can overlap cyclically when projected "upward" onto a computer screen.

In 1984 Dawson [4] showed an example of 12 convex tiles, none of which can be translated "upward" without disturbing the others for any choice of "upward." That is, his example cannot be taken apart with two hands by translating one object away from the rest. In 1988 Natarajan [12] gave an example with 16 thin triangular plates. Already, in 1963, Fejes-Toth and Heppes [6] had given an example of 13 convex objects, 12 tetrahedra surrounding a rhombic dodecahedron, for which no single object can move rigidly (according to an arbitrary isometry) without disturbing the others. In Section 5 we confirm their conjecture that the central dodecahedron is not necessary.

Each of these three examples can be viewed as a sphere tiled with overlapping tiles such that the neighbors of a single tile prevent its motion. Each example can be taken apart by translation with two hands, however, by translating the tiles that cover a certain hemisphere away from the rest. Thus, Natarajan [12] conjectured that every configuration $\mathscr{C}$ of convex objects could be taken apart by translation with two hands: that a proper subset $\mathscr{A} \subset \mathscr{C}$ exists that can be translated to infinity by applying a common translation to the objects in $\mathscr{A}$, without disturbing the objects in the complement $\mathscr{C} \backslash \mathscr{A}$. (No point of $\mathscr{A}$ enters the interior of an object in $\mathscr{C} \backslash \mathscr{A}$ during the translation.)

In this paper we construct counterexamples to this conjecture. Section 3 gives a configuration of six tetrahedra and a proof that it cannot be taken apart by translation with two hands. The construction is based on the symmetries of a tetrahedron (the alternating group $A^{4}$ ). Section 4 shows that this configuration is minimal, in the sense that one object out of any configuration of five convex objects can always be translated to infinity. Section 5 turns to arbitrary isometries and confirms a conjecture that Fejes-Toth and Heppes made about their construction, and Section 6 gives a configuration of 30 objects that cannot be taken apart by applying an isometry to any proper subset. The 30 objects are formed by lacing five copies of the translational counterexample of Section 3 together (based on five subgroups of $A^{4}$ that can be found in the alternating group $A^{5}$ ). The proof of this case is an exhaustive computer check of all nonsymmetric cases, using an analysis technique for positive-force closure grasps in robotics [11], [14] to check for possible infinitesimal rigid motions. 
A short video on the counterexamples was included as part of the Video Review at the Ninth ACM Symposium on Computational Geometry [16]. DEC Systems Research Center was distributing the Video Review in VHS format (NTSC, PAL, or SECAM) as Technical Report 101a and 101b (email: src-report@src.dec.com).

\section{Preliminaries}

We build the counterexamples of Sections 3, 5, and 6 using symmetry groups. This reduces the number of subsets that must be checked for possible disassembly. Because we can use group-theoretic notation to describe the constructions compactly, we use this section to describe our notation.

In brief, we use the symmetry groups of a tetrahedron and a dodecahedron, which are the alternating groups $A^{4}$ and $A^{5}$, respectively. Group elements are represented by rotation matrices and group action on a set of points is simply multiplication of the points by the appropriate rotation matrix.

In more detail, consider a regular tetrahedron, centered at the origin, with vertices $a=(+1,+1,+1), \quad b=(+1,-1,-1), c=(-1,+1,-1)$, and $d=$ $(-1,-1,+1)$. Two of the symmetries of this tetrahedron can be represented by the rotation matrices

$$
R=\left(\begin{array}{rrr}
-1 & 0 & 0 \\
0 & -1 & 0 \\
0 & 0 & +1
\end{array}\right) \quad \text { and } \quad N=\left(\begin{array}{rrr}
0 & +1 & 0 \\
0 & 0 & +1 \\
+1 & 0 & 0
\end{array}\right)
$$

where $R$ rotates tetrahedron abcd by $180^{\circ}$ around the line through segments $\overline{a d}$ and $\overline{b c}$ and $N$ rotates it by $120^{\circ}$ around the line through $a$ and the face $\triangle b c d$. (Multiplying the vertex vectors by the matrices, we see that $a R=d, d R=a$, $b R=c, c R=b$, and that $a N=a, b N=c, c N=d$, and $d N=b$.)

Using the operation of matrix multiplication, we can generate a group by taking products of $N$ and $R$. Because of the identities $R^{2}=I, N^{3}=I$, and $(N R)^{3}=I$, there are 12 distinct matrices that can be formed as products of $N$ and $R$-these are the rotation matrices representing the 12 symmetries of the oriented tetrahedron $a b c d$. Readers familiar with groups may recognize this as the alternating group $A^{4}$, which has a finite presentation [3]:

$$
A^{4}=\left\{N, R \mid N^{3}=I, R^{2}=I,(N R)^{3}=I\right\} .
$$

If we construct an object, we can use the rotation matrices to obtain symmetric objects. As a simple example, the point $a=(1,1,1)$ multiplied by the 12 different rotation matrices gives rise to the four vertices of tetrahedron abcd, with each vertex appearing three times. Using the algebraists' convention of postmultiplying and associating from the left, $a R=d, a R N=b, a R N N=c$, and $a=a N=a N N$ (even though matrices $I \neq N \neq N N$ ). This application of group elements (rotation matrices) to elements of some other space (points in $E^{3}$ ) is an example of a group 
action. The set of all images of a set under a given group action is the orbit of the set. (For rigorous definitions, see a book on group theory or abstract algebra [7].)

In the next section we define a plane through three points $p=(6,5,-2)$, $p R=(-6,-5,-2)$, and $p N=(-2,6,5)$. If we call this plane $\pi$, then the orbit of $\pi$ under the group action of $A^{4}$ contains 12 distinct planes. Plane $\pi N$, for example, passes through $p N, p R N$, and $p N N$.

\section{A Twisted Tetrahedron}

In this section we construct a configuration of six identical convex sticks that cannot be taken apart with two hands using translational motions. Our construction, illustrated in Fig. 1, essentially takes six edges of a tetrahedron, extends them, and gives then a clockwise twist around each vertex, then defines planes separating these edges and creates sticks as the intersections of half-spaces bounded by these planes.

We prove four properties of this construction: First, the sticks defined as intersections of half-spaces are nonempty, bounded convex objects. Second, sticks have disjoint interiors. Third, sticks share two-dimensional contact regions. Fourth, for any proper subset of sticks, every possible translation causes a moving stick to penetrate a stationary one at some contact region.

During the construction, we use the rotation matrices $N$ and $R$ that generate $A^{4}$, the group of symmetries of a tetrahedron, as described in the previous section. We begin with the 12 points formed by $A^{4}$ acting on the point $p=(6,5,-2)$. Because the line segment joining $p$ and $p R$ is invariant under rotation $R$, the orbit of segment $(p ; p R)$ consists of six different segments, each generated twice. (These segments are the extended and twisted edges of a tetrahedron.)

The three points $p=(6,5,-2), p R=(-6,-5,-2)$, and $p N=(-2,6,5)$ define a plane with equation $92+\mathbf{n} \cdot q=0$, where $\mathbf{n}=(35,-42,46)$ is the normal vector to the plane. A point $q$ is said to be in the nonpositive half-space of this plane if $92+\mathbf{n} \cdot q \leq 0$. Notice that symmetries of the tetrahedron, which are pure rotations,
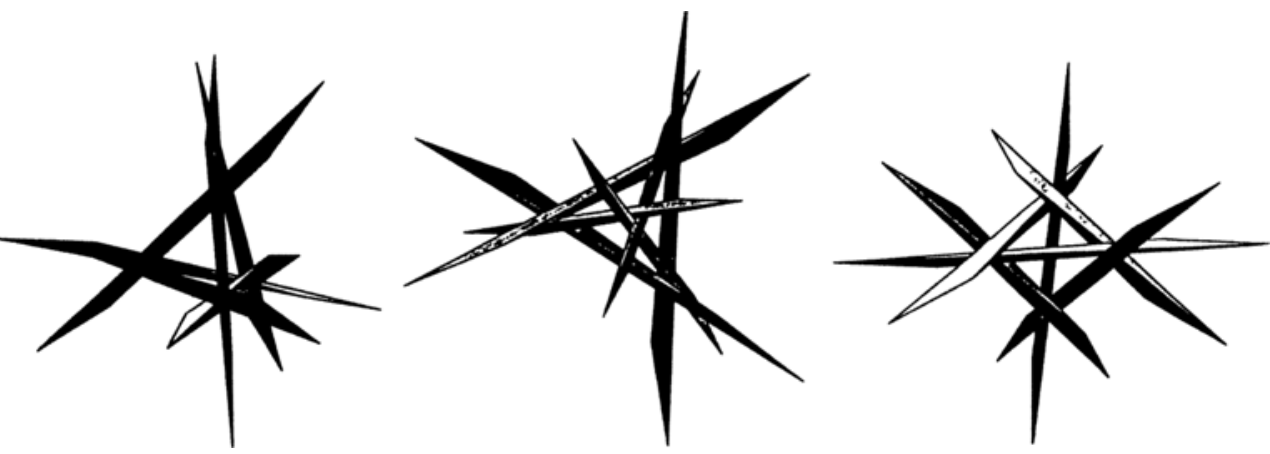

Fig. 1. Three views of six objects that cannot be taken apart by two hands using translations. 
Table 1. The four planes that contain $p$ or $p R$ and define stick $\Xi$.

\begin{tabular}{rrl}
\hline Plane equation for $\Xi$ & Normal vector & \multicolumn{1}{c}{ Points defining the plane } \\
\hline $92+\mathbf{n} \cdot q=0$ & $\mathbf{n}=(35,-42,46)$ & $\{p, p N, P R\}$ \\
$92+\mathbf{n} R \cdot q=0$ & $\mathbf{n} R=(-35,42,46)$ & $\{p R,(p N) R,(p R) R=p\}$ \\
$-92-\mathbf{n} N N \cdot q=0$ & $-\mathbf{n} N N=(42,-46,-35)$ & $\{p N N,(p N) N N=p,(p R) N N\}$ \\
$-92-\mathbf{n} N N R \cdot q=0$ & $-\mathbf{n} N N R=(-42,46,-35)$ & $\{p N N R,(p N) N N R=p R,(p R) N N R\}$ \\
\hline
\end{tabular}

act on the normal vector as they do on points and do not change the constant in a plane equation. Thus, we can name the 12 planes in the orbit of $92+\mathbf{n} \cdot q=0$ by their normal vectors. Four planes in the orbit contain either $p$ or $p R$ (see Table 1.) We can orient these planes so that plugging in the point $q=\left(0,0,-\frac{5}{2}\right)$, for example, gives negative values for all four planes. We call the intersection of the four nonpositive half-spaces the stick $\Xi$.

Lemma 1. The stick $\Xi$, which is the intersection of the nonpositive half-spaces of $92+\boldsymbol{n} \cdot q, 92+n R \cdot q,-92-n N N \cdot q$, and $-92-n N N R \cdot q$, is a nonempty, bounded tetrahedron.

Proof. The intersection is nonempty: $\left(0,0,-\frac{5}{2}\right)$ is in the interior of the nonpositive half-spaces of the four planes. The intersection is also bounded: because the positive linear combination of the normals $35(\mathbf{n}+\mathbf{n} R)+46(-\mathbf{n} N N-\mathbf{n} N N R)=0$, every vector in the subspace spanned by the normals can be written as a positive linear combination of the normals. It can be verified that the normals are not coplanar, so every vector has positive projection on the normal to some plane, and motion along the vector will eventually leave the nonpositive half-space of that plane.

The only nonempty, bounded region defined by four planes is a tetrahedron.

We can apply the group action to $\Xi$ to obtain an orbit with six different sticks, each generated twice.

Lemma 2. The six sticks defined by the group action of $A^{4}$ on $\Xi$ have disjoint interiors.

Proof. Stick $\Xi N$ uses the plane with equation $-92-(\mathbf{n} N N) N \cdot q=-92-\mathbf{n} \cdot q$. Since $\Xi$ uses this plane with the opposite sign, the interiors of $\Xi N$ and $\Xi$ are separated. In the same way, the interiors of $\Xi N R, \Xi N N$, and $\Xi N N R$ are separated from that of $\Xi$.

The only remaining stick is $\Xi N R N N$, which in Fig. 1 is clearly disjoint from $\Xi$. For a numerical proof, it can be checked that the plane $(0,0,1) \cdot q=0$ separates the vertices of the two sticks. 
Next, we observe that sticks that share a plane have a two-dimensional contact. We call such sticks neighbors. Because of symmetries, it is sufficient to check that $\Xi$ and $\Xi N$ are neighbors.

Lemma 3. The intersection of sticks $\Xi$ and $\Xi N$ is a two-dimensional region.

Proof. The point $r=(-3404,-3105,-2975) / 1365$ satisfies $92+\mathbf{n} \cdot r=0$, so $r$ is in the plane shared by sticks $\Xi$ and $\Xi N$. The point $r$ is strictly below the unshared planes of these two sticks - plugging $r$ into the left-hand sides of the plane equations gives strictly negative numbers therefore $r$ is contained in a twodimensional polygon $\Xi \cap \Xi N$.

Theorem 4. Figure 1 illustrates a configuration $\mathscr{C}$ of six tetrahedra that cannot be taken apart by translation with two hands.

Proof. A stationary point in contact with the interior of a face of a moving object rules out all translations with positive projection onto the normal of that face. To show that a proper subset of $\mathscr{C}$ cannot translate infinitesimally, it is enough to show that any direction has positive projection on such a contact normal.

We consider all possible subsets, up to symmetry. Whether a subset or its complement moves depends on one's point of view, so we can focus on subsets of at most three sticks.

The proof of Lemma 1 has already shown that every vector can be expressed as a positive linear combination of the normals of a single stick. Thus, no stick with four stationary neighbors can translate.

Next, suppose that a moving subset $\mathscr{A}$ contains a stick $\Xi$ that has three stationary neighbors. We show that $\mathscr{A}$ can translate only if $\mathscr{A}-\{\Xi\}$ can translate. Let $\mathbf{n}$ be the normal of $\Xi$ at the contact with its moving neighbor and let $\mathbf{n}_{1}, \mathbf{n}_{2}$, and $\mathbf{n}_{3}$ be the normals at stationary contacts. The vector $-\mathbf{n}$ can be expressed as a positive linear combination of $n_{1}, n_{2}$, and $n_{3}$, by Lemma 1 . If we treat $\Xi$ as stationary and find that some translation $t$ is ruled out for $\mathscr{A}-\{\Xi\}$ because $(-n) \cdot t>0$, then $n_{i} \cdot t>0$ for some $n_{i}$ and translation $t$ is also ruled out for $\mathscr{A}$ by motion of $\Xi$ into one of its stationary contacts.

The only possible subsets remaining are three sticks that form a "tripod" or a "triangle"-these subsets are complements. We therefore consider moving the tripod formed by sticks $\Xi, \Xi N$, and $\Xi N N$. The normals at stationary contacts are:

\begin{tabular}{lr}
\hline Stick & \multicolumn{1}{c}{ Contact normal } \\
\hline$\Xi$ & $\mathrm{n} R=(-35,42,46)$ \\
& $-\mathrm{n} N N R=(-42.46,-35)$ \\
$\Xi N$ & $\mathrm{n} R N=(46,-35,42)$ \\
& $-\mathrm{n} N N R N=(-35,-42,46)$ \\
$\Xi N N$ & $\mathbf{n} R N N=(42,46,-35)$ \\
& $-\mathbf{n} N N R N N=(46,-35,-42)$ \\
\hline
\end{tabular}


These normals span the space of all vectors and

$$
31(\mathbf{n} R+\mathbf{n} R N+\mathbf{n} R N N)+53(-\mathbf{n} N N R-\mathbf{n} N N R N-\mathbf{n} N N R N N)=0 .
$$

Therefore, we conclude that no proper subset can be translated without penetrating its stationary complement.

de Bruijn [5], and later Dawson [4], proved that any collection of $n$ star-shaped objects with disjoint interiors can be separated by $n$ hands using translation: For each object, choose a point $q_{i}$ from which object $i$ is star-shaped, then translate object $i$ with the velocity vector $q_{i}-q_{1}$. Since this translation could be accomplished by scaling the entire configuration about $q_{1}$ and then shrinking each object $i$ radially about $q_{i}$ to its original size, there are no intersections during translation. Therefore, $n$ hands-i.e., $n$ distinct motions-are sufficient for convex objects. Natarajan, in private communication, has pointed out that $n / 2$ hands are sufficient for constructions in which convex objects can be partitioned into pairs that are in contact. Each pair becomes a star-shaped set, and de Bruijn's argument applies. Thus, our twisted tetrahedron can be taken apart by translation with three hands.

\section{One of Five Can Translate}

To prove that the construction of the previous section is minimal, we show first that any member of any configuration $\mathscr{C}$ of four or fewer convex objects can be translated to infinity and second that some member of any configuration $\mathscr{C}$ of five convex objects can be translated to infinity.

We call set of planes $\Pi$ a separating set for an object $A \in \mathscr{C}$ if, for any object $B \in \mathscr{C}, B \neq A$, some plane $\pi_{A B} \in \Pi$ separates the interiors of $A$ and $B$. The set $\Pi$ is minimal if no proper subset of $\Pi$ is a separating set for $A$. The region of $A$ with respect to a separating set $\Pi$ is the intersection of half-spaces that contain $A$ defined by planes of $\Pi$. We observe that objects that can be translated to infinity have unbounded regions.

Lemma 5. A member $A$ of a set $\mathscr{C}$ of convex objects with disjoint interiors can be translated to infinity if and only if the region of $A$ is unbounded with respect to some separating set of planes.

Proof. Because the region of $A$ is convex, if it is also unbounded, then it contains infinite rays from $A$. Object $A$ can be translated along such a ray without crossing a plane of the separating set, therefore $A$ can be translated to infinity without encountering another object of $\mathscr{C}$.

If $A$ can be translated to infinity in direction $\alpha$, then let $\hat{A}$ be the union of $A$ and all its translates. $\hat{A}$ is convex and its interior is disjoint from the other objects of the set. For each other object, we can find a plane that separates it from $\hat{A}$-these planes also form a separating set for $A$. The region of $\hat{A}$, which is also the region of $A$, is unbounded. 
The "if" part of this lemma has an easy corollary:

Corollary 6. Any member of a configuration $\mathscr{C}$ of at most four convex objects can be translated to infinity in some direction.

Proof. A minimal separating set for an object $A \in \mathscr{C}$ consists of at most three planes, which divide 3-space into eight unbounded regions.

With difficulty that is disproportionate to the importance of the theorem, we can prove

Theorem 7. Some member of a configuration $\mathscr{C}$ of five convex objects can be translated to infinity.

Proof. We prove this theorem by supposing that there is a counterexample consisting of five disjoint objects and then deriving a contradiction. First we prove that the objects can be assumed to be bounded tetrahedra defined by ten separating planes and that any pair have intersecting faces. We then choose a set $P$ of ten points in these intersection regions: one point common to each pair of tetrahedra. We study the structure of the convex hull $C H(P)$, and prove that it has a ring of five triangular faces on its boundary. Two adjacent triangles must be on the same "side" of this ring; we can use those triangles to show that the objects are not disjoint.

Lemma 8. If there is a counterexample to Theorem 7, then there is one with five bounded tetrahedra $A, B, C, D$, and $E$ defined by the ten planes $\pi_{A B}, \pi_{A C}, \ldots, \pi_{D E}$ that separate pairs. Furthermore, faces defined in the same plane intersect: e.g., $A \cap B$ is a polygon in $\pi_{A B}$.

Proof. Suppose that we are given a configuration $\mathscr{C}$ of five convex objects with disjoint interiors such that no object can be translated to infinity. We make from it a configuration of five tetrahedra.

Choose a mininal set of planes $\Pi$ that is a separating set for each of the objects. Note that $\Pi$ contains at most ten planes: one plane $\pi_{A B}$ for each pair of objects $A$, $B \in \mathscr{C}$. If $\Pi$ contains fewer than ten planes, then some object $X \in \mathscr{C}$ has a separating subset of at most three planes; the region of $X$ is unbounded and Lemma 5 states that $X$ can be translated to infinity. We can therefore assume that every object in $\mathscr{C}$ has a separating subset of four planes of $\Pi$. Since four planes in 3-space define only one bounded region, a tetrahedron, we can further assume that each object is contained in the tetrahedron defined by its four separating planes. We can expand each object to fill its tetrahedron: $\Pi$ remains a separating set for the expanded objects and translation becomes only more difficult.

For each pair of expanded objects (tetrahedra) $A$ and $B$, we show that the intersection $A \cap B$ is a two-dimensional region in the separating plane $\pi_{A B}$. Initially, let $\pi$ equal $\pi_{A B}$. If the interiors of the faces $A \cap \pi$ and $B \cap \pi$ do not 


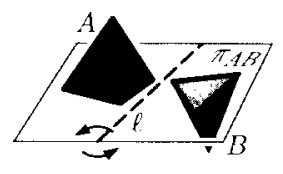

Fig. 2. Faces in $\pi_{A B}$.

intersect, then these faces can be separated in $\pi$ by a line $/$ as in Fig. 2. Rotate $\pi$ around $l$ so that $\pi$ continues to separate $A$ and $B$, until $\pi$ hits the vertex of $A$ or of $B$ that is not in plane $\pi_{A B}$. Suppose, without loss of generality, that $\pi$ hits the vertex of $A$. Then the set $\left\{\pi, \pi_{A C}, \pi_{A D}, \pi_{A E}\right\}$ is a separating set of planes for $A$ that all pass through a common point. In this set the region of $A$ must be unbounded and $A$ can translate to infinity.

We denote the vertex of the tetrahedron $A$ that is $\pi_{A C} \cap \pi_{A D} \cap \pi_{A E}$ by $A_{B}$ because it is opposite the face that intersects $B$. Let $V$ denote the set of all 20 vertices from the five tetrahedra.

As illustrated in Fig. 3 , the $A_{B}$-cone is the region defined by $\pi_{A C}, \pi_{A D}$, and $\pi_{A E}$ that contains $A$; the $A_{B}$-anticone is the region separated from $A$ by $\pi_{A C}, \pi_{A D}$, and $\pi_{A E}$. There are three facts to notice about cones and anticones. First, any plane that cuts the $A_{B}$-cone in a triangle does not intersect the $A_{B}$-anticone, and vice versa. Second, $A$ will be strictly inside the convex hull of any four points chosen one from the interior of each of the four anticones defined by $A$ 's vertices. Third, if the vertex $A_{B}$ is on the convex hull $C H(V)$, then the $A_{B}$-anticone is entirely outside $C H(V)$.

Because tetrahedra $A$ and $B$ intersect in a two-dimensional region in $\pi_{A B}$, we can choose a point $p_{A B} \in A \cap B$ that lies on no planes of $\Pi \backslash\left\{\pi_{A B}\right\}$. For each (unordered) pair of objects $X$ and $Y$, we can choose $p_{X Y}$ in $X \cap Y$ such that the only plane of $I I$ containing $p_{X Y}$ is $\pi_{X Y}$ and $p_{X Y}$ is in general position with respect to the previously chosen points. (No three points collinear, no four points coplanar, and no three defining a plane parallel to a separating plane.) Let $P$ denote the set of all ten points. To proceed toward a contradiction, we investigate the structure of the convex hull $C H(P)$ using the following technical lemma.

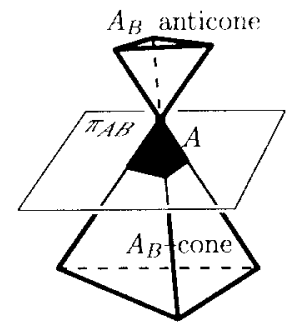

Fig. 3. $A_{B}$-cone and $A_{B}$-anticone. 


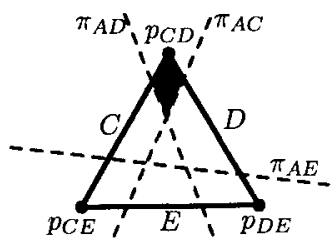

Fig. 4. $\Delta$ cuts the $A_{B}$-cone.

Lemma 9. Suppose that the $A_{B}$-anticone does not intersect $C H(P)$. Then the points $p_{C D}, p_{D E}$, and $p_{C E}$ form a face of $C H(P)$ that lies on the $B$ side of $\pi_{A B}$, is contained in the $B_{A}$-anticone, and whose plane cuts the $B_{A}$-anticone in a triangle.

Proof. Let $\Delta$ denote the triangle defined by $p_{C D}, p_{D E}$, and $p_{C E}$ and let $\gamma$ denote the plane containing $\Delta$. By convexity, the edges of $\Delta$ are contained in tetrahedra $C, D$, and $E$ as labeled in Fig. 4.

By assumption, $p_{C D}$ is not in the $A_{B}$-anticone. Since planes $\pi_{A C}$ and $\pi_{A D}$ separate $A$ and $p_{C D}$, we know that the entire region containing $p_{C D}$ that is defined by $\pi_{A C}$ and $\pi_{A D}$ (shaded in Fig. 4) is on the $A$-side of $\pi_{A E}$. Therefore, $\pi_{A E}$ cuts through the triangle $\Delta$ and separates the region containing $p_{C D}$ from tetrahedron $E$. Similarly, planes $\pi_{A C}$ and $\pi_{A D}$ cut through $\Delta$ and $\Delta$ cuts the $A_{B}$-cone in a triangle as shown in Fig. 4.

Now, we know that in the plane $\gamma$, rays starting from $p_{C D}$ and proceeding through $E$ intersect planes $\pi_{A E}, \pi_{C E}$, and $\pi_{D E}$ before $E$. By Lemma 5 these rays must intersect $\pi_{B E}$ afterward (otherwise $E$ would be in an unbounded region-see Fig. 5). Similar arguments apply to rays through $p_{C E}$ and $D$ and $p_{D E}$ and $C$. Thus, in the plane $\gamma$, the planes $\pi_{B C}, \pi_{B D}$, and $\pi_{B E}$ form a triangle that contain $p_{C D}, p_{D E}$, and $p_{C E}$ as shown in Fig. 5. In other words, the plane $\gamma$ cuts the $B_{A}$-anticone in a triangle that contains $\Delta$.

Since $B$ is a bounded tetrahedron defined by planes $\pi_{A B}, \pi_{B C}, \pi_{B D}$, and $\pi_{B E}$, there is no point in space that is separated from $B$ by all four planes. Therefore, $\Delta$ is on the $B$ side of $\pi_{A B}$.

Because the plane $\gamma$ cuts the $B_{A}$-anticone in a triangle, $\gamma$ does not intersect $B$.

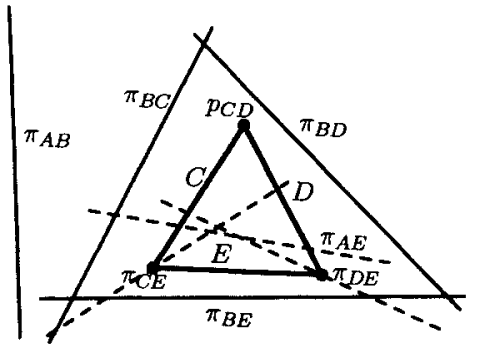

Fig. 5. $\Delta$ is in the $B_{A}$-anticone. 
Moreover, $\gamma$ cuts the $A_{B}$-cone on the $B$ side of the separating plane $\pi_{A B}$. Therefore $\gamma$ does not intersect $A$. Point $p_{A B}$ is common to $A$ and $B$, so both $A$ and $B$ lie strictly to one side of $\gamma$. Since all other points of $P$ lie on $A$ or $B, \gamma$ certifies that $p_{C D}, p_{D E}$, and $p_{C E}$ form a face of $C H(P)$.

As a corollary of this lemma, we can show that if no points of $C H(P)$ are in the $A_{B}$-anticone, then there must be points of $C H(P)$ in several other anticones. For example, at most one anticone from each tetrahedron is free of points of $C H(P)$.

Corollary 10. If the $A_{B}$-anticone is free of points of $C H(P)$, then the $B_{A}$-anticone, other anticones from $A$, and other anticones opposite $B$ must contain points of $P$.

Proof. Suppose the $A_{B}$-anticone is free of points of $C H(P)$. Then Lemma 9 says that $p_{C D} \in C H(P)$ is contained in the $B_{A}$-anticone.

Lemma 9 also says that $p_{C D}$ is on the $E$ side of $\pi_{B E}$, since $p_{C D}$ is in the $B_{A}$-anticone. Therefore, $p_{C D}$ cannot be in the $E_{A}$-anticone and, by the converse of Lemma 9, the $A_{E}$-anticone must intersect $C H(P)$. Similarly, $p_{C E}$ is on the $D$ side of $\pi_{B D}$ and $p_{D E}$ is on the $C$ side of $\pi_{B C}$, so the $A_{D}$-anticone and $A_{C}$-anticone also contain points of $C H(P)$.

Finally, Lemma 9 says that $p_{C D}$ is on the $B$ side of $\pi_{A B}$. Therefore, $p_{C D}$ cannot be in the $B_{C}$-anticone and, using the converse of Lemma 9 once again, the $C_{B}$-anticone contains points of $C H(P)$. Similar arguments say that the $D_{B}$-anticone and $E_{B}$-anticone also contain points of $C H(P)$.

We can now determine that all ten points of $P$ appear on their convex hull, $C H(P)$, as part of a ring of five triangles.

Lemma 11. By relabeling tetrahedra, we can assume that the following five triangles appear as faces of $C H(P): \triangle p_{C D} p_{D E} p_{C E}, \triangle p_{A D} p_{D E} p_{A E}, \triangle p_{A B} p_{B E} p_{A E}, \triangle p_{A B} p_{B C} p_{A C}$, and $\triangle p_{B C} p_{C D} p_{B D}$.

Proof. We show that each tetrahedron has an anticone that is free of points $P$, then argue that Corollary 10 permits only one listing of anticones, up to relabeling.

Notice that if any tetrahedron has points of $P$ in each of its anticones, then that tetrahedron is strictly inside the convex hull $C H(P)$. Now, the convex hull of the tetrahedra, $C H(V)$, has a vertex, which we can label $A_{B}$. Because the $A_{B}$-anticone does not intersect $C H(P)$, Lemma 9 says that points $p_{C D}$ and $p_{D E}$ lie on $C H(P)$. However, this implies that the tetrahedra $C, D$, and $E$ are not strictly inside $C H(P)$ they each must have an anticone free of points of $P$. The corollary implies that these anticones cannot be opposite $B$, so Lemma 9 applied to one of these anticones will show that a point of $B$ also lies on $C H(P)-B$ also has an empty anticone.

Corollary 10 shows that there is one empty anticone per tetrahedron and one of each subscript. With five anticones, this can only occur as a five-cycle $A_{B}, B_{C}, C_{D}$, $D_{E}$, and $A_{E}$ or as a three- and two-cycle. Since two-cycles (e.g., $A_{B}$ and $B_{A}$ ) are 


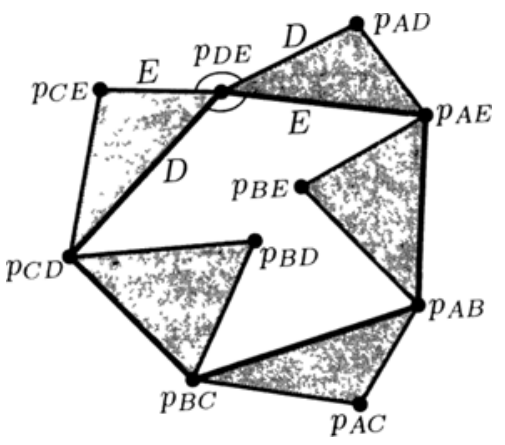

Fig. 6. The ring of faces.

explicitly ruled out by Corollary 10 , we must have the five-cycle, up to relabeling. Applying Lemma 9 to these five empty anticones give the five faces of $C H(P)$.

We can now finish the proof of Theorem 7 by showing that two objects must intersect. As Fig. 6 illustrates, the five triangular faces found by Lemma 11 form a ring on the convex hull $C H(P)$. Since the ring has odd length, there must be two adjacent triangles that lie on the same side of the ring: they are $\triangle p_{C D} p_{D E} p_{C E}$ and $\triangle p_{A D} p_{D E} p_{A E}$ in the figure. However, then the edges incident on the common vertex alternate: coming from objects $D, E, D, E$ in the figure. Because the objects are convex, this alternation means that the interiors of $D$ and $E$ are not disjoint. This contradicts our initial assumption, and establishes the theorem.

\section{The Example of Fejes-Toth and Heppes}

After seeing that not all configurations can be taken apart with two hands using translation, one naturally asks about other rigid motions. As mentioned in the introduction, Fejes-Toth and Heppes [6] have given a configuration of 13 objects -12 tetrahedra packed around a rhombic dodecahedron in which no single object can move by any infinitesimal rigid motion (isometry). They also conjectured that the central dodecahedron was unnecessary. In this section we prove this conjecture as an example of analysis of infinitesimal isometries.

Theorem 12. There is a configuration of 12 tetrahedra with disjoint interiors such that no single tetrahedron can be moved by an infinitesimal isometry.

We begin with our regular tetrahedron abcd from Section 2. A tetrahedral body is defined by the four vertices $72 b, 36 b, 72 c$, and $24(b+d)$. Figure 7 illustrates three of the 12 bodies that are generated by the group action of $A^{4}$.

Stationary contact points on moving faces will rule out certain isometries; we determine which ones using an analysis technique that is common to closure grasps 


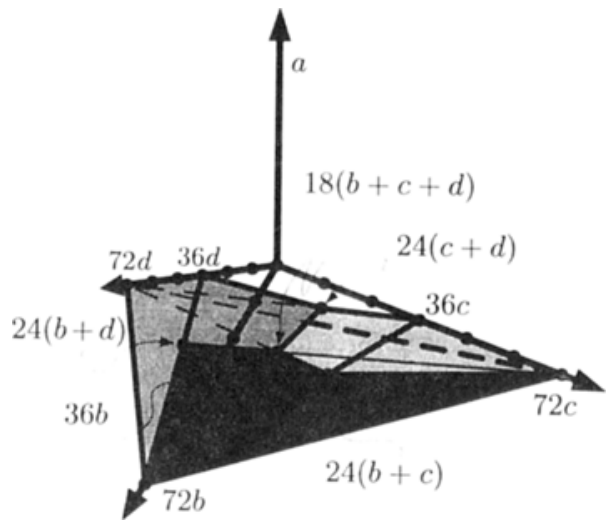

Fig. 7. Three of the 12 bodies.

[11], [14] and assembly-sequence planning [10], [17] in robotics. Any infinitesimal rigid motion can be expressed as translational force and a rotational torque applied to the origin and can be represented as a force/torque vector with six coordinates. If we apply a force at a point $p$, pushing into a face with normal $\mathbf{n}$, then the force and torque applied to the origin is a positive multiple of the force/torque vector $\mathbf{f}=(-\mathbf{n},-\mathbf{n} \times p)$, where $\times$ denotes the three-dimensional vector outer (or cross) product. Therefore, if we can find a set of stationary contact points whose force/torque vectors $\mathbf{f}_{1}, \ldots, \mathbf{f}_{k}$ span the six-dimensional force/torque space by positive linear combinations, then those points immobilize the body any force/torque vector will have positive projection on one of the $\mathbf{f}_{i}$.

Now, to prove that the body with vertices $72 b, 36 b, 72 c$, and $24(b+d)$ cannot move rigidly, we need to find normals and contacts for its faces. Figure 8 shows the faces of this body unfolded into the plane after cutting along each of the three edges

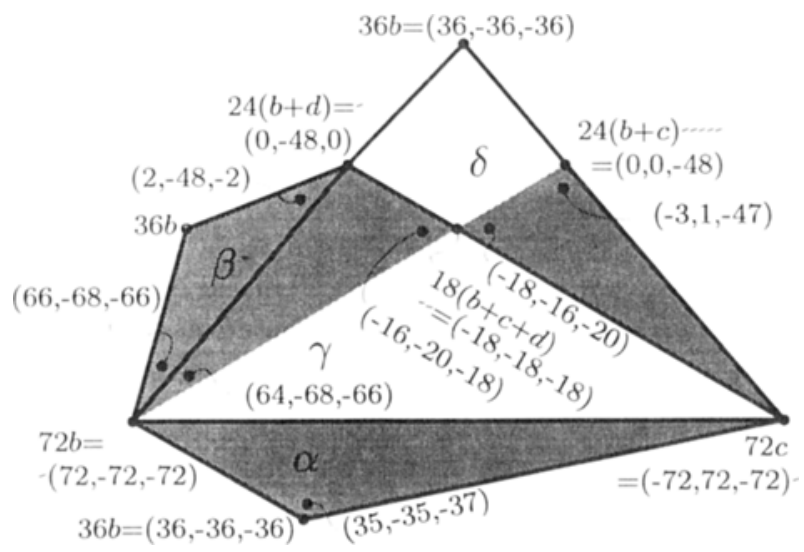

Fig. 8. A body unfolded. 
Table 2. Body vertices.

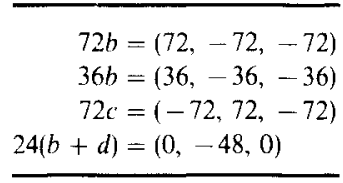

Table 3. Body plane equations.

\begin{tabular}{rlrl} 
& $\alpha:$ & 0 & $=(1,1,0) \cdot q$ \\
$\beta:$ & 0 & $=(1,0,1) \cdot q$ \\
$\gamma:$ & 144 & $=(-3,-3,-2) \cdot q$ \\
$\delta:$ & -144 & $=(2,3,3) \cdot q$ \\
\hline
\end{tabular}

Table 4. Contacts and force/torque vectors for the body.

\begin{tabular}{lll}
\hline Plane & \multicolumn{1}{c}{ Contact } & \multicolumn{1}{c}{ Force/torque vector } \\
\hline$\alpha$ & $(35,-35,-37)$ & $\mathbf{f}_{1}=(1,1,0,-37,37,-70)$ \\
$\beta$ & $(2,-48,-2)$ & $\mathbf{f}_{2}=(1,0,1,68,132,-68)$ \\
$\beta$ & $(66,-68,-66)$ & $\mathbf{f}_{3}=(1,0,1,48,4,-48)$ \\
$\gamma$ & $(64,-68,-66)$ & $\mathbf{f}_{4}=(-3,-3,-2,62,-326,396)$ \\
$\gamma$ & $(-16,-20,-18)$ & $\mathbf{f}_{5}=(-3,-3,-2,14,-22,12)$ \\
$\delta$ & $(-3,1,-47)$ & $\mathbf{f}_{6}=(2,3,3,-144,85,11)$ \\
$\delta$ & $(-18,-16,-20)$ & $\mathbf{f}_{7}=(2,3,3,-12,-14,22)$ \\
\hline
\end{tabular}

incident on vertex $36 b$. From the Cartesian coordinates of the vertices (Table 2) we compute four plane equations, $\alpha, \beta, \gamma$, and $\delta$, with outward-pointing normals (Table 3). The faces of Fig. 8 are labeled by the planes that contain them.

As the shading in Fig. 8 indicates, the whole of the faces in planes $\alpha$ and $\beta$ are in contact with faces of other bodies. In the plane $\gamma$ the portion of the face bounded by $72 b, 24(b+d)$, and $18(b+c+d)$ is in contact; in the plane $\delta$ the portion bounded by $72 c, 24(b+c)$, and $18(b+c+d)$ is in contact. We choose seven contact points from the interiors of these contact regions as listed in Table 4 .

The interested reader can check that these vectors span the six-dimensional force/torque space and that

$$
218400 \mathbf{f}_{1}+71252 \mathbf{f}_{2}+1548 \mathbf{f}_{3}+43927 \mathbf{f}_{4}+101673 \mathbf{f}_{5}+848 \mathbf{f}_{6}+71952 \mathbf{f}_{7}=\mathbf{0} .
$$

Therefore the body cannot move by any infinitesimal rigid motion.

\section{A Twisted Triacontahedral Design}

In this section we form a configuration of 30 identical convex objects that cannot be taken apart with two hands using arbitrary isometries (rigid motions). The name comes from Coffin's book [2], which includes some amazing puzzles based on 30 sticks arranged around the 30 faces of a triacontahedra. Our proof of Theorem 13 relies on computer support.

Theorem 13. Figure 9 illustrates a configuration of 30 convex sticks with disjoint interiors that cannot be taken apart with two hands using arbitrary isometries. 

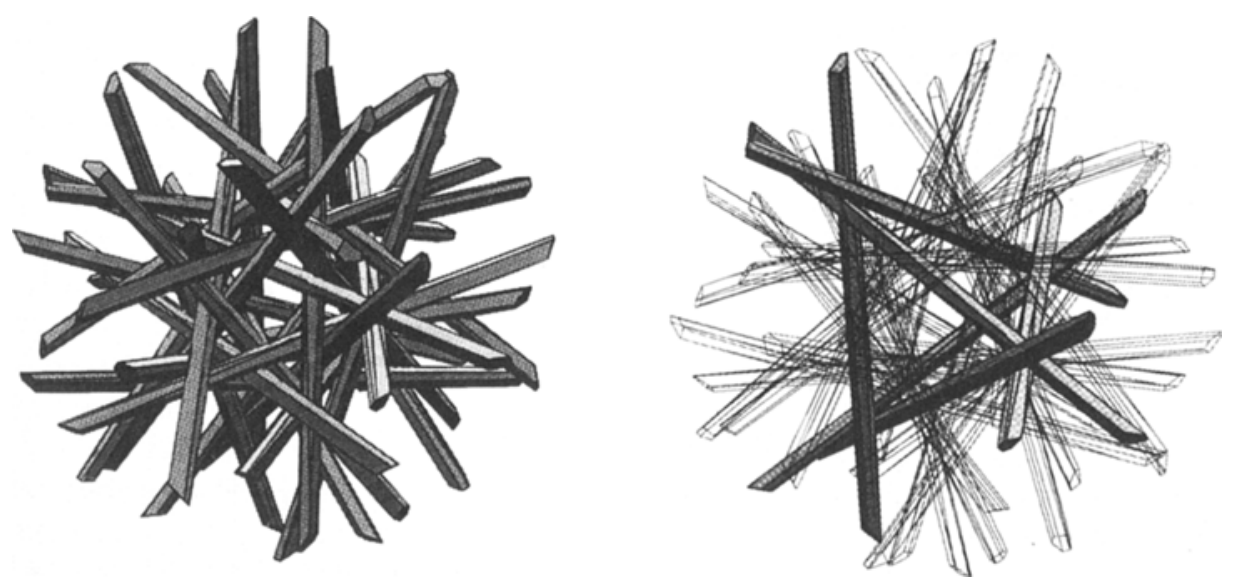

Fig. 9. Thirty objects that cannot be taken apart by two hands using arbitrary isometries.

Our construction laces five copies of the twisted tetrahedron into a dodecahedron - Fig. 9 on the right depicts one as solid among four wireframes. These copies correspond to five subgroups of $A^{4}$ in $A^{5}$, which is the group of symmetries of a dodecahedron. By judiciously choosing separating planes, we get sticks that share two-dimensional contacts with ten neighbors.

Let $\varphi=(1+\sqrt{5}) / 2$ denote the golden ratio and define rotation matrices

$$
F=\frac{1}{2}\left(\begin{array}{ccc}
\varphi & 1 & 1 \\
-1 & 1 & \varphi \\
1 & \varphi & \\
\varphi & -\varphi & 1
\end{array}\right) \text { and } E=\frac{1}{2}\left(\begin{array}{ccc}
-1 & 1 & \varphi \\
1 & \varphi & \\
\varphi & -\varphi & 1 \\
\varphi & 1 & 1 \\
\varphi
\end{array}\right) \text {. }
$$

$F$ and $E$ generate $A^{5}$, the group of symmetries of a regular dodecahedron:

$$
A^{5}=\left\{F, E \mid F^{5}=I, E^{2}=I,(E F)^{3}=I\right\} .
$$

(Given the dodecahedron whose vertices are points in the orbit of $(1,1,1)$ under $A^{5}$, $F$ rotates by $2 \pi / 5$ about a line through the center of a face; $E$ rotates by $\pi$ about a line through the center of an edge.) The group elements

$F E F E=N=\left(\begin{array}{rrr}0 & +1 & 0 \\ 0 & 0 & +1 \\ +1 & 0 & 0\end{array}\right) \quad$ and $\quad E F F E F=R=\left(\begin{array}{rrr}-1 & 0 & 0 \\ 0 & -1 & 0 \\ 0 & 0 & +1\end{array}\right)$

are the generators of the $A^{4}$ subgroup that we used in Section 3. The group elements $\left\{I=F^{0}, F^{1}, F^{2}, F^{3}, F^{4}\right\}$ each lie in different cosets of this subgroup. (That 
Table 5. Planes separating $(p ; p R)$ from neighbors.

\begin{tabular}{crl}
\hline Segment & Plane equation & Contact point \\
\hline $\mathbf{n}_{1}=(64,-79,97) / 3$ & & \\
$\mathbf{c}_{1}=(-0.5280-0.9834 \varphi$, & $0.5265-1.5313 \varphi,-0.8826$ & $-0.5983 \varphi)$ \\
$(p N ; p R N)$ & $-161 / 3=\mathbf{n}_{1} \cdot q$ & $\mathbf{c}_{1}$ \\
$(p N R ; p R N R)$ & $-161 / 3=\mathbf{n}_{1} R \cdot q$ & $\mathbf{c}_{1} R$ \\
$(p N N ; p R N N)$ & $161 / 3=\mathbf{n}_{1} N N \cdot q$ & $\mathbf{c}_{1} N N$ \\
$(p N N R ; p R N N R)$ & $161 / 3=\mathbf{n}_{1} N N R \cdot q$ & $\mathbf{c}_{1} N N R$ \\
\hline $\mathbf{n}_{2}=(-55+25 \varphi, 58-26 \varphi, 64+27 \varphi) / 2$ & \\
$\mathbf{c}_{2}=(0.2337-0.7339 \varphi, 0.5216-0.7734 \varphi, 0.0636$ & $-1.1568 \varphi)$ \\
$(p E F ; p R E F)$ & $-4-57 \varphi=\mathbf{n}_{2} \cdot q$ & $\mathbf{c}_{2}$ \\
$(p E F R ; p R E F R)$ & $-4-57 \varphi=\mathbf{n}_{2} R \cdot q$ & $\mathbf{c}_{2} R$ \\
$(p E F E F ; p R E F E F)$ & $4+57 \varphi=\mathbf{n}_{2} E F E F \cdot q$ & $\mathbf{c}_{2} E F E F$ \\
$(p E F E F R ; p R E F E F R)$ & $4+57 \varphi=\mathbf{n}_{2} E F E F R \cdot q$ & $\mathbf{c}_{2} E F E F R$ \\
\hline $\mathbf{n}_{3}=(-25-30 \varphi, 30+36 \varphi,-19+19 \varphi) / 2$ & \\
$\mathbf{c}_{3}=(0.7462-1.1956 \varphi,-1.9418+0.7462 \varphi,-1.1956$ & $-0.4493 \varphi)$ \\
$(p E ; p R E)$ & $0=\mathbf{n}_{3} \cdot q$ & $\mathbf{c}_{3}$ \\
$(p E R ; p R E R)$ & $0=\mathbf{n}_{3} R \cdot q$ & $\mathbf{c}_{3} R$ \\
\hline
\end{tabular}

is, we get distinct copies of $A^{4}$ in $A^{5}$ by starting with $F^{i}$ and multiplying by $N$ 's and $R$ 's.)

We construct sticks in a manner similar to that in Section 3: Begin with the 60-point orbit of $p(6,5,-2)$ under the action of $A^{5}$. There are 30 line segments in the orbit of segment $(p ; p R)$, each generated twice. Then define planes separating $(p ; p R)$ from neighboring segments and intersect their half-spaces to form one stick. Each stick is defined by ten separating planes, which are grouped into three types in Table 5. Four planes separate $(p ; p R)$ from neighbors within its $A^{4}$ subgroup, four planes separate it from neighbors in an $A^{4}$ subgroup generated by $R$ and $E F$, and two other bisecting planes are defined.

By numerical tests similar to those performed in Section 3, we can see that these planes form nonempty, bounded sticks with disjoint interiors. Each stick has two-dimensional contact with its ten neighbors, as shown in Fig. 10. (In this figure

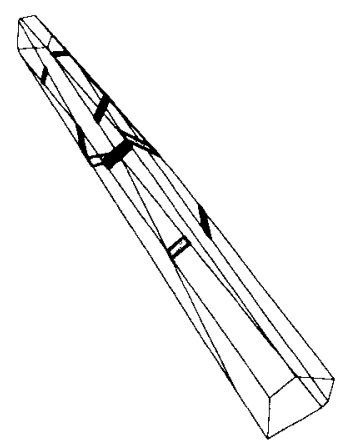

Fig. 10. Stick contacts. 
and in Fig. 9 the ends of the sticks have been clipped so that the interesting part of the construction may be seen.) We choose one point from each contact region (the average of the vertices) and form the force/torque vectors that apply to the origin when a positive force acts on each contact of each stick. Note that although decimal approximations to the contact vectors are listed in Table 4 for compactness, all these computations can be done exactly in a rational field extended to include the golden ratio $\varphi$ using symbolic algebra packages such as Maple and Mathematica. (We used both.)

We consider possible infinitesimal motions of a subset of sticks by a linear programming formulation of the analysis of the previous section: Let $A$ be the $6 \times k$ matrix of force/torque vectors where a moving object is in contact with a stationary point. Nondegenerate basic feasible solutions [1], [13] of the seven linear equations $A \mathbf{x}=\mathbf{0}$ and $\sum_{1 \leq i \leq k} x_{i}=1$ express the origin as a positive linear combination of seven force/torque vectors that span the space. If we show that this linear program is feasible for each subset of sticks, then we have shown that the sticks cannot be taken apart by any two-handed rigid motion. (The fact that seven is sufficient follows from Caratheodory's theorem [9], [11] and that our force/torque vectors are in sufficiently general position. Otherwise we might have to go to 12 and use Steinitz's theorem.)

There are $2^{30}$ subsets to analyze; we can use a 30 -bit integer to represent each subset. By manipulating these bits, we can avoid analyzing symmetric cases, complementary cases, and cases in which some moving stick has nine or ten stationary neighbors. This reduces the number of subsets to $2^{21.8}$. If we weaken the result to show that no motion is infinity is possible (rather than that no infinitesimal motion is possible), then we can reduce the number of subsets to $2^{16.8}$ by eliminating subsets with a moving triangle that is linked with a stationary triangle or 4-cycle. Solving the linear-programming problems for these subsets (using a revised simplex algorithm that stores only the inverse of the current basis and not the entire tableau [1], [13]) takes 7 minutes on an IBM RS6000/560, including the reduction. Because these reductions also make proving program correctness more difficult, we wind up not using them on the final run of the program, which takes about 25 hours. We also ran the program on two other architectures, at least one of which had a different floating-point implementation.

We should mention that, even with no pivoting, the condition numbers of all matricies that arose were bounded by $10^{6}$ using the $L_{\infty}$ norm. We ran a second check with pivoting on subsets for which the bound on the condition number became larger than $2 \times 10^{4}$. The actual condition numbers in these cases were less than 3 .

A general polynomial-time algorithm could be implemented to analyze a configuration by computing the arrangement that the hyperplanes normal to the contact force/torque vectors induce on the five-dimensional sphere of unit force/ torque vectors in 6-space. Within each cell a unique blocking graph can be defined, with a directed edge from object $A$ to $B$ if contact with $A$ prevents $B$ from moving according to force/torque vectors in the cell. No subset can move infinitesimally iff all blocking graphs are strongly connected. (See [17] for more detail.) In our case, however, the 150 hyperplanes form an arrangement with $2^{34}$ cells, and further reductions require tricky programming. 


\section{Conclusion}

The constructions in this paper are further evidence, if any were needed, that favorite techniques and tools of computational geometry in the plane break down in 3-space.

Many mathematical and algorithmic questions remain to be answered: Exactly how many hands are necessary to take apart any configuration of convex objects? A specific configuration? If one is told how many hands are sufficient for a specific configuration, how does one efficiently compute a motion? An entire disassembly sequence?

\section{Acknowledgments}

We thank Simon Kahan and Dale Rolfsen for helping to debug proofs that one of five objects can translate, Randy Wilson for his paper on the polynomial-time disassembly algorithm and other references to the literature on mechanical assembly planning, and the referees for their comments.

\section{References}

1. V. Chvátal. Linear Programming. Freeman, San Francisco, CA, 1983.

2. S. T. Coffin. The Puzzling World of Polyhedral Dissections. Oxford University Press, Oxford, 1991.

3. R. H. Crowell and R. H. Fox. Introduction to Knot Theory. Blaisdell, New York, 1965.

4. R. Dawson. On removing a ball without disturbing the others. Mathematics Magazine, 57(1):27-30, 1984.

5. N. G. de Bruijn. Problems 17 and 18. Nieuw Archief voor Wiskunde, 2:67, 1954. Answers in Wiskundige Opgaven met de oplossingen, 20:19-20, 1955.

6. L. Fejes-Toth and A. Heppes. Uber stabile Körpersysteme. Compositio Mathematica, 15(2): 119-126, 1963.

7. J. B. Fraleigh. A First Course in Abstract Algebra. Addison-Wesley, Reading, MA, 1982.

8. L. J. Guibas and F. F. Yao. On translating a set of rectangles. Proceedings of the 12th Annual ACM Symposium on Theory of Computing, pages 154-160, 1980.

9. P. McMullen and G. C. Shephard. Convex Polytopes and the Upper Bound Conjecture. Cambridge University Press, Cambridge, 1971.

10. L. S. H. d. Mello. Computer-Aided Mechanical Assembly Planning. Kluwer, Boston, 1991.

11. B. Mishra, J. T. Schwartz, and M. Sharir. On the existence and synthesis of multifinger positive grips. Algorithmica, 2:541-558, 1987.

12. B. K. Natarajan. On planning assemblies. Proceedings of the Fourth Annual ACM Symposium on Computational Geometry, pages 299-308, 1988.

13. C. H. Papadimitriou and K. Steiglitz. Combinatorial Optimization: Algorithms and Complexity. Prentice-Hall, Englewood Cliffs, NJ, 1982.

14. J. Pertin-Troccaz. Grasping: A state of the art. In O. Khatib, J. J. Craig, and T. Lozano-Perez, editors, The Robotics Review 1, pages 71-98. MIT Press, Cambridge, MA, 1989.

15. F. P. Preparata. Planar point location revisited. International Journal of Foundations of Computer Science, 1(1):71-86, 1990.

16. J. Snoeyink. Video: Objects that cannot be taken apart with two hands. Proceedings of the Ninth Annual ACM Symposium on Computational Geometry, page 405, 1993. Video Review of Computational Geometry also available as DEC SRC Report 101. 4:39 animation.

17. R. H. Wilson and T. Matsui. Partitioning an assembly for infinitesimal motions in translation and rotation. IEEE International Conference on Intellegent Robots and Systems, pages 1311-1318, 1992. 